\title{
A FELICIDADE EM PESSOAS COM DOENÇA MENTAL: UMA REVISÃO INTEGRATIVA DA LITERATURA
}

\author{
| Paulo Seabra ${ }^{1}$; Alexandra Sarreira-Santos ${ }^{2}$; Lurdes Medeiros-Garcia ${ }^{3}$; Alexandre Costa ${ }^{4}$; Idália Cardoso ${ }^{5}$ Amélia S. Figueiredo ${ }^{6}$ |
}

\section{RESUMO}

CONTEXTO: A forma como cada pessoa vive e sente satisfação com a sua experiência de vida, tem naturalmente uma dimensão subjetiva mas reveladora de aspetos intrínsecos da dimensão humana. A felicidade pode ser um conceito agregador dessas dimensões embora de difícil definição para pessoas com ou sem doença mental.

OBJETIVO: Identificar a perceção de felicidade em pessoas com doença mental, os fatores que contribuem para aumentar ou menorizar essa perceção.

MÉTODOS: Revisão Integrativa da Literatura. Pesquisa em bases de dados internacionais, realizada em agosto de 2016. Foi aplicado um conjunto de descritores e critérios de inclusão. Obteve-se uma amostra final de 7 estudos, publicados entre 2006-2014.

RESULTADOS: Não se encontraram definições aprofundadas do conceito expressas pelos participantes. Emerge como aproximação ao conceito a capacidade para interação social ou enquanto fenómeno intrinsecamente relacionada com a religiosidade. A felicidade depende pouco de eventos externos e reforça-se com fatores duradouros e de uma dimensão mais interior e predeterminada previamente à doença. Os fatores que contribuem são essencialmente de ordem pessoal, familiar, suporte social alargado e emocional. Os que contribuem para menorizar a felicidade são de ordem pessoal, efeitos secundários da medicação, escassez de suporte social alargado, disfunção afetiva e emocional. A família não surgiu enquanto fator associado a menor felicidade.

CONCLUSÕES: A felicidade está interligada à vivência social e ao bem-estar, à resiliência, a fatores protetores internos da própria pessoa.

\section{PALAVRAS-CHAVE: Felicidade; Transtornos mentais; Qualidade de vida}

\section{RESUMEN}

"La felicidad en las personas con enfermedad mental: Una revisión integradora de la literatura"

CONTEXTO: La forma que cada persona vive y siente la satisfacción con su experiencia de vida, es por supuesto una dimensión subjetiva, pero revelador de aspectos intrínsecos de la dimensión humana. La felicidad puede ser un concepto unificador de estas dimensiones, aunque difícil definir para personas con o sin enfermedad mental.

OBJETIVO: Identificar la percepción de felicidad en personas con enfermedad mental, los factores que contribuyen a aumentar o disminuir esta percepción.

METODOLOGÍA: Revisión Integradora de Literatura. Búsqueda en bases de datos internacionales efectuada en Agosto, 2016. Se ha aplicado un conjunto de descriptores y criterios de inclusión. Esto produce una muestra final de 7 estudios publicados entre 2006-2014.

RESULTADOS: No se ha encontrado definiciones en profundidad expresadas por los participantes. Surge como aproximación al concepto la capacidad para la interacción social o como fenómeno estrechamente ligado a la religiosidad. La felicidad depende menos de eventos externos y se refuerza con factores de larga duración y de dimensión más interior, predeterminada antes de la enfermedad. Los factores que contribuyen son personales, familiares, de apoyo social extendido y emocional. Aquellos que contribuyen para reducirla son factores personales, efectos secundarios de la medicación, falta de apoyo social extendido, disfunción afectiva e emocional. La familia no surgió como factor asociado a la felicidad inferior.

CONCLUSIONES: La felicidad está conectada a la vida social y al bienestar, la capacidad de recuperación, factores protectores internos de la persona.

\section{DESCRIPTORES: Felicidad; Enfermedades mentales; Calidad de vida}

\begin{abstract}
“The happiness of people with mental illness: An integrative literature review"

BACKGROUND: The way each person lives and feels satisfaction with their life experience, naturally has a subjective but revealing dimension of intrinsic aspects of the human dimension. Happiness can be an aggregating concept of these dimensions although difficult to define for people with or without mental illness.

AIM: To identify the happiness perception in people with mental illness, the factors that contribute to increase or reduce this perception.

METHODS: Integrative Literature Review. Research in international databases, held in August 2016. A set of descriptors and inclusion criteria were used. A final sample of 7 studies, published between 2006-2014, was obtained.

RESULTS: There were no in-depth definitions of the concept expressed by participants. It emerges as an approach to the concept, the capacity for social interaction or as an intrinsically phenomenon related to religiosity. Happiness depends little on external events and is reinforced by enduring factors and a more inward and predetermined dimension prior to illness. The factors that contribute are essentially personal, family, extended social support and emotional. Those that contribute to lower happiness are personal, side effects of medication, lack of broad social support, affective and emotional dysfunction. The family did not arise as a factor associated with less happiness.
\end{abstract}

CONCLUSION: Happiness is intertwined with social experience and well-being, with resilience, with person internal protective factors.

\section{KEYWORDS: Happiness; Mental disorders; Quality of life}

1 Doutor em Enfermagem; Professor Auxiliar Convidado na Universidade Católica Portuguesa, pauloseabra@ics.lisboa.ucp.pt

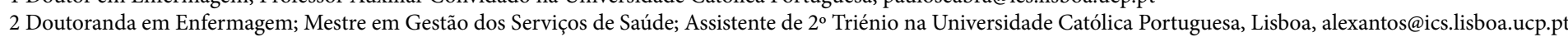
3 Doutoranda em Enfermagem; Mestre em Ciências da Educação; Assistente de 2º Triénio na Universidade Católica Portuguesa, Lisboa, mgarcia@ics.lisboa.ucp.pt

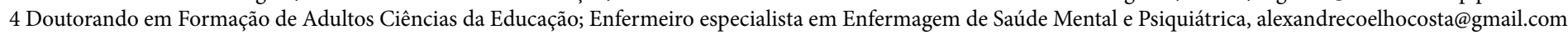

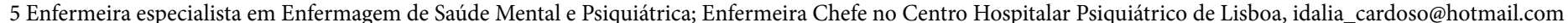

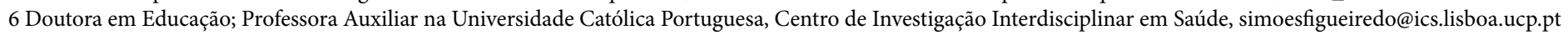




\section{INTRODUÇÃO}

A desigualdade verificada no acesso aos cuidados de saúde e a vivência com "doença mental", seja de forma institucionalizada, ou autónoma, pode levar-nos a uma reflexão sobre a auto perceção do estado de saúde das pessoas com doença mental. Nesse sentido, e tendo em conta, aspetos mais subjetivos relacionados com a saúde mental e com a qualidade de vida, e no que isso tem de intrínseco com a forma como cada um vive e sente satisfação com a sua experiência de vida, surgiu, a reflexão sobre a perceção de "felicidade" em pessoas com doença mental. A procura da felicidade é um objetivo humano fundamental pois mais felicidade está associada a mais saúde (Agid et al., 2012; World Healt Organization [WHO], 2011).

Para se compreender a perceção de felicidade em pessoas com doença mental, alguns aspetos são determinantes. No que se refere às pessoas com esquizofrenia, é evidenciada maior dificuldade em obter melhores resultados face aos sintomas negativos, em contraponto dos positivos, e é evidenciada a diminuição progressiva das capacidades sociais e ocupacionais desde a jovem idade adulta (Agid et al., 2012). Para estas dificuldades, intervenções promotoras de reabilitação, de relações sociais e de sentido de vida, permitem maior perceção da felicidade, fundamental para o recovery. É preciso identificar barreiras para experienciar a felicidade, implementar estratégias para que possam assumir a responsabilidade pela sua vida, procurar experiências que lhes tragam felicidade, pois estas promovem esperança e o estabelecimento de uma identidade positiva (Buckland, Schepp, \& Crusoe, 2014). A fase da doença surge como fator importante pois os mais jovens tendem a ter mais expetativas e esperança que os doentes há mais tempo (Kaplan, Salzar, \& Brusilovskiy, 2012). A felicidade é uma emoção básica caracterizada por um estado emocional positivo, com sentimentos de bem-estar e de prazer, associados à perceção de sucesso e à compreensão coerente e lúcida do mundo (Ferraz, Tavares, e Zilberman, 2007). Desempenha um papel importante na dimensão física, mental/bem-estar, nas relações sociais, nas emoções e cognição, no desenvolvimento moral e no desempenho (Martens, 2006), no entanto, não existe uma definição consensual, (Palmer, Martin, Depp, Glorioso, \& Jeste, 2014). É um fenômeno predominantemente subjetivo, mais subordinado a traços psicológicos, socioculturais e até genéticos, do que estritamente a fatores ou eventos isolados e externamente determinados (Ferraz et al., 2007; Palmer et al., 2014).
Pessoas com a mesma doença não percecionam felicidade da mesma maneira (Buckland et al., 2014). Para alguns autores, a felicidade não é um bom indicador para a qualidade de vida das pessoas com doença mental porque, o seu humor tende a refletir estados psicológicos alterados e não, circunstâncias objetivas das suas vidas, embora, as dúvidas, sobre se as pessoas conseguem julgar as suas vidas adequadamente, pela distorção possível ao nível afetivo e cognitivo, se estendam à população em geral, pois não se investigam estas perceções quando as pessoas estão em estado psicótico (Bergsma, Veenhoven, Have, \& de Graaf, 2011). Por outro lado, na população em geral, uma percentagem elevada dos que não se sentem felizes nos últimos dias, têm psicopatologia (Bergsma \& Veenhoven, 2011a; Bergsma et al., 2011),ou seja, é um indicador importante em qualquer população. Apesar desta subjetividade, a felicidade tem sido defendida como um indicador a ter em conta nas decisões futuras sobre a vida das pessoas (Loewenstein \& Ubel, 2008; WHO, 2011) pois, é considerada por muitos, como uma efetiva avaliação de qualidade de vida, sendo coisas diferentes. A felicidade tem uma dimensão cognitiva (Veenhoven, 2009) e para alguns autores, a qualidade de vida é uma condição prévia, mais do que uma experiência intrínseca de felicidade (Agid et al., 2012). Tem-se verificado, que a satisfação com a vida e a felicidade, não é muito baixa nas pessoas com doença mental, o que pode sugerir que essa perceção não se deve a eventos exteriores e circunstanciais, que a falta de insight aumenta a perceção de uma forma não justificável ou ainda, que a sua perceção não esteja consonante com o resto da população (Bergsma et al., 2011). Surge muitas vezes, em pessoas com esquizofrenia, valores positivos, e até equivalentes à população em geral (Palmer et al., 2014). As pessoas com esquizofrenia tendem a ter os mesmos objetivos de vida que tinham antes da doença, o que provoca sofrimento pela diminuição da funcionalidade (Agid et al., 2012).

$\mathrm{O}$ facto de muitas pessoas com doença mental viverem em ambientes protegidos como residências com algum apoio social, surge associado a mais felicidade que naqueles que vivem sozinhos ou com as suas famílias (Buckland et al., 2014; Palmer et al., 2014). Importa a reflexão sobre as intervenções que combatem um dos fatores que mais contribuem para perceção negativa da felicidade, a anedonia (Agid et al., 2012). Na população em geral a felicidade pode ainda ser um fator preditivo e promotor de bem-estar, pois tem sido associada à diminuição de possíveis perturbações (Agid et al., 2012; Bergsma et al., 2011; Homayouni, 2010). 
Quadro 2 - Conjugações de Pesquisa

\begin{tabular}{|l|l|}
\hline $\begin{array}{l}\text { Plataforma eletrónica de } \\
\text { base de dados }\end{array}$ & Fórmula de pesquisa \\
\hline B-On & $\begin{array}{l}\text { TITLE (happiness) AND SUB- } \\
\text { JECT TERMS (mental disorders) } \\
\text { OR (mental health) OR (psy- } \\
\text { chiatry) OR (mental illness) OR } \\
\text { (mental well-being) OR (quality } \\
\text { of life) OR (emotion) OR (elderly) } \\
\text { OR (rehabilitation) OR (revovery) } \\
\text { OR (satisfaction) }\end{array}$ \\
\hline EBSCO & $\begin{array}{l}\text { TITLE (happiness) AND AB- } \\
\text { STRACT (mental disorders) OR } \\
\text { (mental illness) OR (recovery) OR } \\
\text { (psychiatry) } \\
\text { TITLE (happiness) OR (well- } \\
\text { being) OR (life satisfaction) } \\
\text { AND ABSRACT (psychiatry) OR } \\
\text { (inpatient) }\end{array}$ \\
\hline BVS & $\begin{array}{l}\text { TITLE (happiness) AND AB- } \\
\text { STRACT (mental disorders) } \\
\text { OR (mental illness) OR (mental } \\
\text { health) OR (recovery) }\end{array}$ \\
\hline
\end{tabular}

Para a seleção dos estudos seguimos as guidelines PRISMA para a identificação, seleção, elegibilidade e inclusão dos estudos na RIL (Moher, Liberati, Tetzlaff, Altman, \& Group, 2009). Cada artigo foi analisado por dois investigadores, de forma independente, com o objetivo de averiguar a concordância na seleção. Naqueles em que não havia concordância, o artigo era revisto por um terceiro investigador para se tomar uma decisão. Com a conjugação dos descritores e operadores referidos no Quadro 2, apurou-se uma amostra de 193 estudos, da qual se obteve uma amostra final de 7 que foram analisados (Fluxograma 1).

Fluxograma 1 - Processo de seleção dos artigos

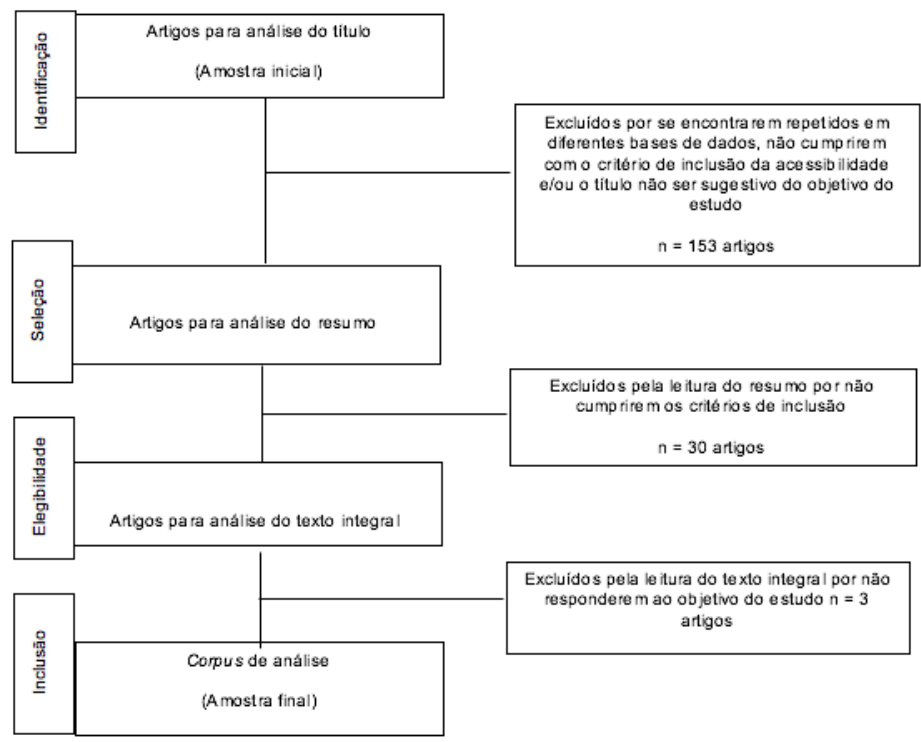

Elaboramos uma grelha de caracterização para descriminar os elementos mais relevantes de cada artigo, ou seja, uma síntese narrativa: título, autores, ano de publicação, país, caracterização dos participantes, desenho, instrumentos, perceção de felicidade, e por fim, os fatores que contribuem para elevar essa perceção e fatores que minimizam a perceção.

\section{RESULTADOS}

O país onde se publicaram mais estudos foi a Holanda (quatro). Os estudos foram publicados entre 2006 e 2014, embora não se encontrasse entre 2007-2009 e 2015-2016. A perceção de felicidade em pessoas com doença mental surge naturalmente acompanhada em muitos estudos, por um conjunto vasto de conceitos que permitem analisar a forma como as pessoas vivem no seu dia-a-dia (qualidade de vida, bem-estar, satisfação, etc.). Sistematizamos em quadros a informação mais relevante (Quadros 3 a 9).

Quadro 3 - Dados do Estudo 1

\begin{tabular}{|c|c|}
\hline No & Referência do artigo \\
\hline 1 & $\begin{array}{l}\text { "Defining Happiness for Young Adults } \\
\text { with Schizophrenia: A Building Block } \\
\text { for Recovery" (Buckland et al., 2014) }\end{array}$ \\
\hline $\begin{array}{l}\text { Participantes / Intervenção } \\
\text { /País }\end{array}$ & Método / Recolha de dados \\
\hline $\begin{array}{l}\text { Participantes: } 11 \\
\text { Média de idades } 27 \text { [23-25]. } \\
\text { Habilitações: } 6 \text { com ensino } \\
\text { secundário, } 7 \text { com estudos } \\
\text { acima do ensino secundário. } \\
\text { Intervenção a que são sujeitos: } \\
\text { Serviços de saúde mental } \\
\text { comunitária e grupos de } \\
\text { autoajuda. } \\
\text { País: EUA }\end{array}$ & $\begin{array}{l}\text { Tipo de estudo: Estudo piloto de- } \\
\text { scritivo. Método misto. Amostra de } \\
\text { conveniência intencional por "bola } \\
\text { de neve". Análise interpretativa de } \\
\text { conteúdo quantificando perceções. } \\
\text { Três entrevistas semiestruturadas a } \\
\text { cada participante (intervalo temporal } \\
\text { de } 6 \text { semanas), com o referencial do } \\
\text { conceito de reabilitação e utilizando o } \\
\text { inquérito apreciativo. }\end{array}$ \\
\hline \multicolumn{2}{|c|}{ Felicidade - Definição / Características definidoras / Dimensões } \\
\hline \multicolumn{2}{|l|}{ - } \\
\hline \multicolumn{2}{|c|}{ Fatores que contribuem para a felicidade: } \\
\hline \multicolumn{2}{|c|}{$\begin{array}{l}\text { Sentir ajuda de outras pessoas (categoria social e dimensão relacional); } \\
\text { Desenhar (categoria cognitiva, propriedade criativa, dimensão indi- } \\
\text { vidual); frequentar serviço religioso (categoria espiritual e social); ter } \\
\text { namorada (categoria social); ter amigos (relações sociais), manter laços } \\
\text { familiares; ser tratado como normal. }\end{array}$} \\
\hline \multicolumn{2}{|c|}{ Barreiras / fatores que contribuem para menorizar a felicidade: } \\
\hline \multicolumn{2}{|c|}{$\begin{array}{l}\text { Medo (relacionado com a insegurança); isolamento; medicação (apesar } \\
\text { de valorizada, provoca dificuldades em: estar alerta, iniciativa, contro- } \\
\text { lar peso, compromisso com atividades agradáveis, ter um sentimento } \\
\text { positivo em relação à sexualidade e autoestima, diminuição da libido, } \\
\text { não ser considerado "normal" (estigma e auto estigma)). }\end{array}$} \\
\hline
\end{tabular}


Quadro 4 - Dados do Estudo 2

\begin{tabular}{|l|l|}
\hline No & Referência do artigo \\
\hline 2 & $\begin{array}{l}\text { "Do They Know How Happy They Are? On the } \\
\text { Value of Self-Rated Happiness of People With a } \\
\text { Mental Disorder" (Bergsma et al., 2011) }\end{array}$ \\
\hline $\begin{array}{l}\text { Participantes / } \\
\text { Intervenção /País }\end{array}$ & Método / Recolha de dados \\
\hline $\begin{array}{l}\text { Participantes: 1135. } \\
\text { Intervenção a } \\
\text { que são sujeitos: } \\
\text { nenhum programa } \\
\text { clínico. }\end{array}$ & $\begin{array}{l}\text { Tipo de estudo: Prospetivo com avaliação aos 0, } \\
\text { Instrumentos quantitativos: Perceção da felici- }\end{array}$ \\
País: Holanda & $\begin{array}{l}\text { Health Questionnaire (GHQ-12)); Lócus de } \\
\text { Control (Mastery Scale); Neuroticismo (Gron- } \\
\text { inger Neuroticism Questionnaire); Autoestima } \\
\text { (Rosenberg Self-esteem Scale) }\end{array}$ \\
\hline \multicolumn{2}{|l|}{ Felicidade - Definição / Características definidoras } \\
\hline - \\
\hline \multicolumn{2}{|l|}{ Fatores que contribuem para a felicidade: } \\
\hline $\begin{array}{l}\text { - Não ter perturbação mental (nem como estado num determinando } \\
\text { momento, nem como diagnóstico único ou associado); Menores } \\
\text { problemas psicológicos; Mais autoestima, estabilidade emocional, ter } \\
\text { controlo e satisfação com a sua vida, recreação social, sentir-se com } \\
\text { energia, satisfação com a sua família; Receber uma remuneração pelo } \\
\text { trabalho. }\end{array}$ \\
\hline Barreiras / fatores que contribuem para menorizar a felicidade: \\
\hline $\begin{array}{l}\text { - Ter perturbação mental (perturbação do humor, da ansiedade e abuso } \\
\text { de substâncias); Duplo diagnóstico; Maior número de episódios de } \\
\text { perturbação mental (mais crónicos). }\end{array}$ \\
\hline
\end{tabular}

Quadro 5 - Dados do Estudo 3

\begin{tabular}{|c|c|}
\hline No & Referência do artigo \\
\hline 3 & $\begin{array}{l}\text { "Wellness within illness: Happiness in schizo- } \\
\text { phrenia" (Palmer et al., 2014) }\end{array}$ \\
\hline $\begin{array}{l}\text { Partic } \\
\text { Interv }\end{array}$ & Método / Recolha de dados \\
\hline $\begin{array}{l}\text { Participantes: } 1^{\circ} \\
\text { grupo: } 72 \text { utentes } \\
\text { ( } 54,2 \% \text { mulheres); } \\
\text { Média de idade } \\
50,1 \text { anos [23-70]; } \\
\text { média de } 24,4 \text { anos } \\
\text { de doença; Média } \\
\text { de idade de apare- } \\
\text { cimento da doença } \\
25,8 \text { anos; } \\
2^{\circ} \text { grupo: } 64 \text { (não } \\
\text { doentes); média de } \\
\text { idade de } 50,7 \text { anos; } \\
\text { Intervenção a que } \\
\text { são sujeitos: Segui- } \\
\text { dos em estruturas } \\
\text { de apoio ambu- } \\
\text { latório } \\
\text { País: EUA }\end{array}$ & $\begin{array}{l}\text { Tipo de estudo: Comparativo, transversal, cor- } \\
\text { relacional } \\
\text { Instrumentos: Percepção de felicidade: (4 } \\
\text { questões / CES-D); Severidade da psicopatologia } \\
\text { (Positive and Negative Symptoms Scales / Brief } \\
\text { Symptom Inventory-Anxiety subscale (BSI-A)); } \\
\text { Qualidade de vida (SF 36); Impacto das comorbi- } \\
\text { lidades (Cumulative Illness Rating Scale for Ge- } \\
\text { riatrics (CIRS-G)); Perceção de stress (Perceived } \\
\text { Stress Scale); Resiliência (Hardy-Gill Resilience } \\
\text { Scale (HGRS)); Otimismo (Life Orientation } \\
\text { Test-Revised (LOT-R)); Suporte social (Duke } \\
\text { Social Support Index (DSSI)); Lócus de controlo } \\
\text { (Personal Mastery Scale (PMS)); Atitude face ao } \\
\text { envelhecimento (Philadelphia Geriatric Center } \\
\text { (PGC)); Experiência espiritual (Brief Multidi- } \\
\text { mensional Measure of Religiousness/Spirituality } \\
\text { (BMMRS)); Cognição (Telephone Interview for } \\
\text { Cognitive Status-Modified) }\end{array}$ \\
\hline
\end{tabular}

Felicidade - Definição / Características definidoras

\section{Fatores que contribuem para a felicidade:}

- Fatores psicossociais (Melhor perceção da qualidade de vida; maiores níveis de resiliência no que se refere ao traço e a eventos de vida, otimismo e melhor lócus de controlo).

Barreiras / fatores que contribuem para menorizar a felicidade:

- Perceção de maior stress.
Quadro 6 - Dados do Estudo 4

\begin{tabular}{|l|l|}
\hline$N^{\circ}$ & Referência do artigo \\
\hline 4 & $\begin{array}{l}\text { “The happiness of people with a } \\
\text { mental disorder in modern society” } \\
\text { (Bergsma \& Veenhoven, 2011b) }\end{array}$ \\
\hline $\begin{array}{l}\text { Participantes / Intervenção } \\
\text { /País }\end{array}$ & Método / Recolha de dados \\
\hline $\begin{array}{l}\text { Participantes: 7076. Idade } \\
\text { Intervenção a que são sujeitos: } \\
\text { nenhuma } \\
\text { País: Holanda }\end{array}$ & $\begin{array}{l}\text { Tipo de estudo: Quantitativo, trans- } \\
\text { (1 item do SF 36) }\end{array}$ \\
\hline Felicidade - Definição / Características definidoras \\
\hline - & \multicolumn{2}{|l}{} \\
\hline Fatores que contribuem para a felicidade: \\
\hline - Fatores de suporte psicossocial \\
\hline Barreiras / fatores que contribuem para menorizar a felicidade: \\
\hline - Ter doença mental
\end{tabular}

Quadro 7 - Dados do Estudo 5

\begin{tabular}{|l|l|}
\hline No & Referência do artigo \\
\hline 5 & $\begin{array}{l}\text { "Happiness and Related Remission in } \\
\text { Patients with Antisocial Personality } \\
\text { Disorder" (Martens, 2006) }\end{array}$ \\
\hline $\begin{array}{l}\text { Participantes / Intervenção } \\
\text { /País }\end{array}$ & Método / Recolha de dados \\
\hline $\begin{array}{l}\text { Participantes: 1 } \\
\text { Intervenção a que é sujeito: } \\
\text { Unidade de psiquiatria forense } \\
\text { País: Holanda }\end{array}$ & $\begin{array}{l}\text { Tipo de estudo: Estudo de caso } \\
\text { Instrumentos: - }\end{array}$ \\
\hline Felicidade - Definição / Características definidoras \\
\hline - Ter capacidades para interação social \\
\hline Fatores que contribuem para a felicidade: \\
\hline $\begin{array}{l}\text { - Aumento da capacidade / atitudes emocionais e desenvolvimento } \\
\text { moral; Acabar o ensino básico; Ajuda neurobiológica para o controlo } \\
\text { de impulsos e terapia cognitiva; Aumentar as competências sociais } \\
\text { (para menorizar isolamento); Relaxamento e privacidade; Bem-estar } \\
\text { espiritual. }\end{array}$ \\
\hline Barreiras / fatores que contribuem para menorizar a felicidade: \\
\hline $\begin{array}{l}\text { - Tédio, solidão (tornaram-no primitivo, violento e selvagem); Isola- } \\
\text { mento social. } \\
\text { - Atitudes desviantes e disfunção socio emocional (desconfiança, } \\
\text { mente fechada, falta de empatia, indiferença). }\end{array}$ \\
\hline
\end{tabular}

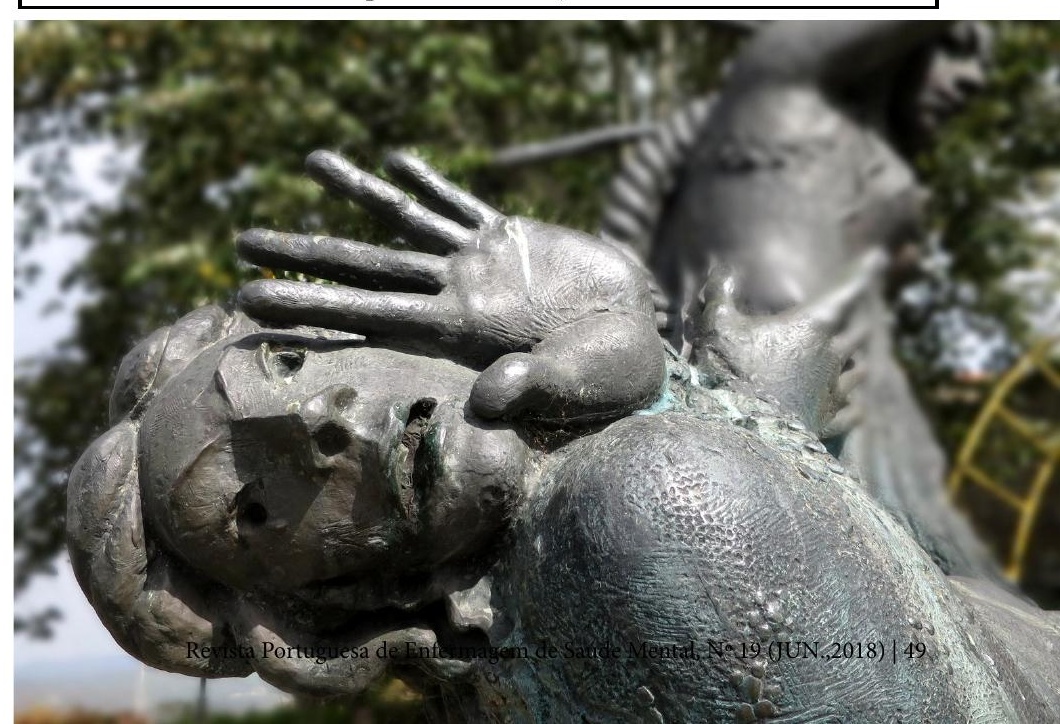


Quadro 8 - Dados do Estudo 6

\begin{tabular}{|l|l|}
\hline No & Referência do artigo \\
\hline 6 & $\begin{array}{l}\text { "A Survey on the role of religious orienta- } \\
\text { tion, mental health and happiness in the senile } \\
\text { people" (Homayouni, 2010) }\end{array}$ \\
\hline $\begin{array}{l}\text { Participantes / } \\
\text { Intervenção /País }\end{array}$ & Método / Recolha de dados \\
\hline $\begin{array}{l}\text { Participantes: } 80 \\
\text { Intervenção: Não } \\
\text { referem } \\
\text { País: Irão }\end{array}$ & $\begin{array}{l}\text { Tipo de estudo: Correlacional; amostra random- } \\
\text { izada. } \\
\text { Instrumentos: Orientação religiosa (Allport's } \\
\text { religious orientation scale (AROS)); Felicidade } \\
\text { (Argyle's happiness inventory (AHI)); Saúde } \\
\text { Mental (General Health Questionnaire (GHQ)). }\end{array}$ \\
\hline Felicidade - Definição / Características definidoras \\
\hline - Associado à religiosidade. \\
\hline Fatores que contribuem para a felicidade: \\
\hline $\begin{array}{l}\text { - Ter maior religiosidade intrínseca contribui para a esperança e felici- } \\
\text { dade em todos os momentos da vida. }\end{array}$ \\
\hline Barreiras / fatores que contribuem para menorizar a felicidade: \\
\hline - Componentes da doença mental \\
\hline
\end{tabular}

Quadro 9 - Dados do Estudo 7

\begin{tabular}{|c|c|}
\hline No & Referência do artigo \\
\hline 7 & $\begin{array}{l}\text { "Happiness in first-episode schizophrenia" (Agid } \\
\text { et al., 2012) }\end{array}$ \\
\hline $\begin{array}{l}\text { Participantes / } \\
\text { Intervenção /País }\end{array}$ & Método / Recolha de dados \\
\hline $\begin{array}{l}\text { "Happiness in first- } \\
\text { episode schizophre- } \\
\text { nia" (Agid et al., } \\
2012 \text { ) } \\
\text { Participantes: } 31 \\
\text { (doentes), Média } \\
\text { de idade de } 24,4 \\
\text { anos [18-35]; } 29 \\
\text { (controlos) } \\
\text { Intervenção: Uni- } \\
\text { dade de } 1^{\circ} \text { episódio } \\
\text { de esquizofrenia } \\
\text { País: Canadá }\end{array}$ & $\begin{array}{l}\text { Tipo de estudo: Misto. Comparativo, random- } \\
\text { izado, de autorrelato } \\
\text { Instrumentos: Sintomas da esquizofrenia (Posi- } \\
\text { tive and Negative Syndrome Scale for Schizo- } \\
\text { phrenia (PANSS) / Clinical Global Impression } \\
\text { scale (CGI)) ; Depressão (Calgary Depression } \\
\text { Scale (CDS)); Insigth (Schedule for Assessment } \\
\text { of Insight (SAI)); Cognição (Brief Assessment } \\
\text { of Cognition in Schizophrenia, version } 3.0 \\
\text { (BACS)), Felicidade (Subjective Happiness Scale } \\
\text { (SHS); Satisfação com a vida (Satisfaction with } \\
\text { Life Scale (SWLS); Funcionalidade (Social Func- } \\
\text { tioning Scale (SFS) }\end{array}$ \\
\hline \multicolumn{2}{|c|}{ Felicidade - Definição / Características definidoras } \\
\hline \multicolumn{2}{|l|}{-} \\
\hline \multicolumn{2}{|c|}{ Fatores que contribuem para a felicidade: } \\
\hline \multicolumn{2}{|c|}{$\begin{array}{l}\text { - Mais satisfação com a vida, melhor funcionamento social e ocupacio- } \\
\text { nal; Performance independente e relações interpessoais. }\end{array}$} \\
\hline \multicolumn{2}{|c|}{ Barreiras / fatores que contribuem para menorizar a felicidade: } \\
\hline$-\mathrm{De}$ & egativos. \\
\hline
\end{tabular}

\section{DISCUSSÃO}

Os estudos foram realizados na Holanda (três), nos EUA (dois), Irão e Canadá (um), maioritariamente de natureza quantitativa. Quanto aos participantes, as amostras são díspares, desde 1 até 7076 participantes.
Os estudos onde as amostras têm maior dimensão, são de base populacional alargada, com apuramento daqueles que se podiam incluir nos critérios de perturbação mental.

O estudo com mais pessoas identificadas com doença mental foi o estudo de Palmer e colaboradores (Palmer et al., 2014) no qual foram entrevistados 72 doentes de unidades de apoio ambulatório. Em apenas dois estudos, verificamos que a idade média dos participantes era inferior a 30 anos (Agid et al., 2012; Buckland et al., 2014) e estes, estavam a ser acompanhados em unidades de apoio a pessoas com esquizofrenia. Nesta faixa etária, os dados apontam perceções numa fase inicial da doença, associadas a mais esperança e espectativas, com repercussão positiva na sua qualidade de vida e funcionalidade (Kaplan et al., 2012).

Sobre a perceção de felicidade das pessoas com doença mental, a contribuição dos estudos para a definição do conceito e as suas características definidoras, é reduzida.

Em nenhum dos estudos, se encontra uma definição aprofundada do conceito, expressa pelos participantes. Em apenas um dos estudos (com a perceção de um participante) a felicidade é definida como ter "capacidade para interação social" (Martens, 2006). Outro, não definindo o conceito, evidencia que felicidade é intrinsecamente relacionada com a religiosidade (Homayouni, 2010). O facto de os resultados não nos aproximarem de uma definição conceptual, surge na linha da dificuldade identificada noutros estudos (Ferraz et al., 2007) mas, dá suporte aos que defendem que felicidade depende menos de eventos efémeros e externos mas reforça-se com fatores duradouros e de uma dimensão mais interior e predeterminada previamente à doença (Palmer et al., 2014).

$\mathrm{Na}$ análise interpretativa, relativa aos fatores que contribuem para perceção da felicidade, é clara a abordagem na dualidade social (Dubar, 2006; Goodson, 2008). Assim, os artigos sustentam os resultados entre duas transações, uma subjetiva, mais relacionada com o self, ou com o individuo na relação consigo próprio, na forma de se capacitar para a relação com os outros e outra, relacional, em múltiplos contextos sociais de trabalho, família, religião etc.

Sobre os fatores que contribuem para aumentar a perceção de felicidade, sistematizamos em diferentes dimensões. 
$\mathrm{Na}$ dimensão pessoal: não ter perturbação mental, ter menos problemas psicológicos, mais autoestima (Bergsma et al., 2011), estabilidade emocional (Bergsma et al., 2011; Martens, 2006), controlo e satisfação com a sua vida (Agid et al., 2012; Bergsma et al., 2011), sentir-se com energia (Bergsma et al., 2011), mais resiliência, mais otimismo e locus de controlo (Palmer et al., 2014), bem-estar espiritual e desenvolvimento moral (Martens, 2006), relaxamento (Martens, 2006), religiosidade intrínseca (Homayouni, 2010), atividades de base recreativa como desenhar (Buckland et al., 2014). $\mathrm{Na}$ dimensão interpessoal / relacional apuramos fatores de três categorias. Fatores relacionados com a família, como a manutenção de laços efetivos (Bergsma et al., 2011; Buckland et al., 2014). Fatores relacionados com suporte social alargado: sentir ajuda por parte de outras pessoas (Buckland et al., 2014), suporte psicossocial profissional (Bergsma \& Veenhoven, 2011b), ajuda neurobiológica para o controlo de impulsos e terapia cognitiva (Martens, 2006), ser tratado como normal (Bergsma \& Veenhoven, 2011b; Buckland et al., 2014), ter amigos (Buckland et al., 2014), interação social e ocupacional (Agid et al., 2012), receber uma remuneração pelo trabalho (Bergsma et al., 2011), treino de capacidades sociais, estudos básicos, privacidade (Martens, 2006).

Fatores a nível afetivo: ter namorada (Buckland et al., 2014).

O contributo para a felicidade emerge essencialmente na dimensão interpessoal, tal como noutros estudos, associado a um suporte alargado (Ferraz et al., 2007; WHO, 2011).

Sobre os fatores que contribuem para menorizar a felicidade, utilizando as mesmas categorias, destaca-se na dimensão pessoal: a medicação que apesar de valorizada, surge associada a várias dificuldades (estar alerta, ter iniciativa, controlar peso, compromisso com atividades agradáveis, sentimento positivo em relação à sexualidade e autoestima, diminuição da libido). A toma da medicação surge associada ainda ao medo de não ser considerado "normal" (Buckland et al., 2014). Os outros fatores identificados foram, ter perturbação mental ou duplo diagnóstico associado (Bergsma \& Veenhoven, 2011b; Bergsma et al., 2011; Homayouni, 2010), cronicidade da perturbação mental (Bergsma et al., 2011), perceção de maior stress (Palmer et al., 2014), depressão, sintomas negativos (Agid et al., 2012).
$\mathrm{Na}$ dimensão interpessoal / relacional, surgiram fatores relacionados com suporte social alargado nomeadamente o medo (relacionado com a insegurança), solidão e isolamento (Buckland et al., 2014; Martens, 2006), ausência de atividades ocupacionais e disfunção social (Martens, 2006).

Fatores a nível afetivo: disfunção emocional (Martens, 2006).

Não se apuraram fatores a relacionados com a família. Sobre os aspetos que menorizam a felicidade, evidenciam-se mais aspetos do domínio pessoal, que interpessoal, aproximando esta discussão do que alguns autores defendem, que a perceção de felicidade tem maioritariamente uma essência pessoal, individual, mais da forma como a pessoa vivencia os acontecimentos de vida, e menos de eventos pontuais e exteriores (Ferraz et al., 2007; Palmer et al., 2014). O acesso ou a falta de apoio igualmente não emergiram como fatores promotores de menor felicidade (Bergsma et al., 2011), embora a sintomatologia ou mesmo a patologia mental é fator determinante para esta menor perceção (Buckland et al., 2014).

Sistematizando os estudos apresenta valores muitos semelhantes de felicidade e satisfação com a vida em geral, embora ligeiramente superiores naqueles sem doença (Bergsma et al., 2011; Martens, 2006; Palmer et al., 2014), apontando para o facto de esta diferença não estar na psicopatologia mas em factos da vida, diminuindo sim, quando é menor a resiliência (Palmer et al., 2014).

Esta resiliência surge como determinante para mais saúde mental, promotora de bem-estar e protetora para aqueles que têm doença mental (Aburn, Gott, \& Hoare, 2016).

Por vezes, conclui-se que a falta de insight pode levar a assinalar valores injustificáveis, mas não há consenso sobre esta afirmação. Enquanto fator protetor para agudização ou cronicidade da pessoa com perturbação, a felicidade pode ser um fator preditivo pois, contribui para diminuir essa possibilidade, contribui para o recovery (Agid et al., 2012; Bergsma et al., 2011; Martens, 2006).

Verifica-se igualmente que é nas pessoas com perturbação do humor, que a felicidade é normalmente mais baixa (Bergsma \& Veenhoven, 2011b). 


\section{CONCLUSÃO}

A diferença da perceção de felicidade entre pessoas com ou sem perturbação mental é, em muitos estudos, pequena e sem significado estatístico, embora, nesta RIL se apure diferenças entre $2,7 \%$ e os $27 \%$, superior naqueles sem perturbação. As condições necessárias para a felicidade também se assemelham, o que deve ser enquadrado na perspetiva que a diferença não está na psicopatologia, mas em factos da vida. Em alguns estudos não se verificam diferenças consoante variáveis socio demográficas, duração da doença, severidade dos sintomas positivos e negativos, função física, comorbilidades e funcionamento cognitivo. A felicidade aumenta a capacidade social, emocional e moral. As pessoas com doença mental sentem-se mais felizes se tiverem as características associadas a uma boa saúde mental, têm uma perceção positiva da felicidade.

Sobre os fatores que condicionam de forma negativa a perceção da felicidade, salientam-se as transações subjetivas e os fatores inerentes à própria doença, como a medicação, os sintomas negativos, o estigma e a autoestima. Apesar do número reduzido de estudos sobre a temática, os dados apontam para que mesmo com doença, os que vivem na comunidade tem valores superiores de felicidade.

Esta RIL contribui para o debate acerca do estudo de um fenómeno, subjetivo e de difícil definição em qualquer grupo populacional. A felicidade está interligada à vivência social e ao bem-estar, à resiliência, a fatores protetores internos da própria pessoa. Defende-se que a intervenção profissional deve promover melhor interação social, recursos associados a realização pessoal com o próprio, apoio familiar e emocional, apoio medicamentoso mas, principalmente promover suporte social alargado. Suporte social não muito diferente de qualquer outro grupo populacional sem doença mental.

Sobre a fiabilidade dos estudos sobre este conceito subjetivo, devido a falta de insight que em algumas situações podem levar as pessoas a assinalar valores injustificáveis, estes achados sugerem-nos que nos doentes, $\mathrm{o}$ funcionamento cognitivo e o insight, não tiveram efeito nos ratings de felicidade. Aliás, os dados apontam para que não seja necessário excluir as pessoas com perturbação, dos estudos da felicidade na população em geral, porque as medidas podem ser as mesmas.

São limitações desta RIL a dificuldade de sistematizar dados de alguns dos estudos principalmente relacionados com a percentagem dos participantes com doença mental.
Os instrumentos utilizados foram de tal maneira díspares, que é difícil fazer comparação de dados. Não se apuraram estudos sobre esta temática em pessoas com doença crónica de evolução prolongada e institucionalizadas.

\section{REFERÊNCIAS BIBLIOGRÁFICAS}

Aburn, G., Gott, M., \& Hoare, K. (2016). What is resilience? An integrative review of the empirical literature. Jounal of Advanced Nursing, 72(5), 980-1000. doi: $10.1111 /$ jan. 12888

Agid, O., McDonald, K., Siu, C., Tsoutsoulas, C., Wass, C., Zipursky, R. B., Foussias, G., \& Remington, G. (2012). Happiness in first-episode schizophrenia. Schizophrenia Research, 141(1), 98-103. doi: 10.1016/j. schres.2012.07.012

Bergsma, A., \& Veenhoven, R. (2011a). The happiness of people with a mental disorder in modern society. Psychology of Well-Being: Theory, Research and Practice, 1(1), 2. doi: 10.1186/2211-1522-1-2

Bergsma, A., \& Veenhoven, R. (2011b). The happiness of people with a mental disorder in modern society. Psychology of Well-Being: Theory, Research and Practice, 1(1), 2. doi: 10.1186/2211-1522-1-2

Bergsma, A., Veenhoven, R., Have, M., \& de Graaf, R. (2011). Do they know how happy they are? On the value of self-rated happiness of people with a mental disorder. Journal of Happiness Studies, 12(5), 793-806. doi: 10.1007/s10902-010-9227-5

Buckland, H., Schepp, K., \& Crusoe, K. (2014). Defining happiness for young adults with schizophrenia: A building block for recovery. Archives of Psychiatric Nursing, 27(5), 235-240. doi: 10.1016/j.apnu.2013.07.002

Dubar, C. (2006). A crise das Identidades: A Interpretação de uma mutação. Porto: Afrontamento.

Ferraz, R., Tavares, H., e Zilberman, M. (2007). Felicidade: Uma revisão. Revista de Psiquiatria Clínica, 34(5), 234-242.

Goodson, I. (2008). Conhecimento e vida profissional estudos sobre educação e mudança. Porto: Porto Editora. 
Homayouni, A. (2010). A survey on the role of religious orientation, mental health and happiness in the senile people. In E. Psychiatry (ed.), 18th European Congress of Psychiatry (Vol. 25, p. 745). Munich: Elsevier. doi: 10.1016/S0924-9338(10)70739-2

Kaplan, K., Salzar, M., \& Brusilovskiy, E. (2012). Community participation as a predictor of recovery - oriented outcomes among emerging and mature adults with mental illnesses. Psychiatric Rehabilitation Journal, 35(3), 219-229.

Loewenstein, G., \& Ubel, P. (2008). Hedonic adaptation and the role of decision and experience utility in public policy. Journal of Public Economics (92), 1795-1810.

Martens, W. (2006). Happiness and related remission in patients with antisocial personality disorder. Annals, Winter, 16-22.

Moher, D., Liberati, A., Tetzlaff, J., Altman, D. G., \& Group, T. P. (2009). Preferred reporting items for systematic reviews and meta-analyses: The PRISMA statement. PLOS Medicine, 6(7). doi: 10.1371/journal. pmed. 1000097
Palmer, B. W., Martin, A. S., Depp, C. A., Glorioso, D. K., \& Jeste, D. V. (2014). Wellness within illness: Happiness in schizophrenia. Schizophrenia Research, 159(1), 151-156. doi: 10.1016/j.schres.2014.07.027

Veenhoven, R. (2009). How do we assess how happy we are? In A. Dutt, \& B. Radcliff (eds.), Happiness, economics and politics (pp. 45-49). Northampton: Edward Elger.

Whittemore, R., \& Knafl, K. (2005). The integrative review: Updated methodology. Jounal of Advanced Nursing, 52(5), 546-553.

World Health Organization. (2011). The happiness effect. Bulletin of the World Health Organization (89), 246-247. doi: 10.2471/BLT.11.020411

\section{AGRADECIMENTOS}

A equipa de investigação teve a colaboração do Sr. Enf. ${ }^{\circ}$ Luís Galhanas e da Sra. Enf. ${ }^{a}$ Paula Arvela, do Centro Hospitalar Psiquiátrico de Lisboa, na revisão do artigo. 